\title{
Novo Coronavírus (2019-nCoV): Análise da magnitude nos dois primeiros meses da epidemia
}

RESUMO | Objetivo: Analisar a magnitude do Novo Coronavírus (COVID-19) no Estado de São Paulo (ESP) nos dois primeiros meses da epidemia a partir da confirmação do primeiro caso. Método: Estudo ecológico, descritivo, considerando os casos confirmados de COVID-19 captados pelo Centro de Vigilância Epidemiológica do ESP para o período de 26 de fevereiro a 26 de abril de 2020. Resultado: Verificou-se que o município de São Paulo apresentou maior número de casos (13989) e óbitos (1172). No entanto, não foi o município que apresentou os maiores indicadores de saúde da COVID-19 (magnitudes: taxa de incidência, mortalidade e letalidade). Conclusão: É provável que tais resultados se devam à falta de testagem do COVID-19 nos municípios do Estado. O potencial da epidemia, ainda, é particularmente preocupante, dado ao grande número de pessoas potencialmente suscetíveis ao COVID19 e a magnitude da mesma que extrapola os indicadores mundiais em algumas localidades.

Palavras-chaves: Infecções por coronavírus; Epidemiologia descritiva; Estudo ecológico.

ABSTRACT | Objective: To analyze the magnitude of the New Coronavirus (COVID-19) in the State of São Paulo (SSP) in the first two months of the epidemic after the confirmation of the first case. Method: Ecological, descriptive study, considering the confirmed cases of COVID-19 captured by the SSP Epidemiological Surveillance Center for the period from February 26 to April 26, 2020. Result: It was found that the municipality of São Paulo presented a greater number of cases (13,989) and deaths $(1,172)$. However, it was not the municipality that presented the highest magnitude for health indicators of COVID-19 incidence rate, mortality and lethality. Conclusion: Um explanation for such results is the lack of testing of COVID-19 in the municipalities studied. The potential of the epidemic is still of particular concern, given the large number of people potentially susceptible to COVID19 and the magnitude of the epidemic that extrapolates world indicators in some locations.

Keywords: Coronavirus infections; Descriptive epidemiology; Ecological study.

RESUMEN I Objetivo: Analizar la magnitud del nuevo coronavirus (COVID-19) en el estado de São Paulo (ESP) en los primeros dos meses de la epidemia después de la confirmación del primer caso. Método: Estudio ecológico descriptivo, considerando los casos confirmados de COVID-19 capturados por el Centro de Vigilancia Epidemiológica ESP para el período del 26 de febrero al 26 de abril de 2020. Resultado: Se encontró em el municipio de São Paulo un mayor número de casos (13989) y defunciones (1172). Sin embargo, no fue el municipio el que presentó las más altas magnitudes para los indicadores de salud de COVID-19 tasa de incidencia, mortalidad y letalidad. Conclusión: És probable que tales resultados se deban a la falta de pruebas de COVID-19 en los municipios del Estado. El potencial de la epidemia sigue siendo motivo de especial preocupación, dada la gran cantidad de personas potencialmente susceptibles a COVID19 y la magnitud de la epidemia que extrapola los indicadores mundiales en algunos lugares.

Palavras claves: Infecciones por coronavirus; Epidemiología descriptiva; Estudio ecologico.

\section{Roudom Ferreira Moura}

Enfermeiro, Doutorando do Programa de Epidemiologia da Faculdade de Saúde Pública - Universidade de São Paulo, Técnico do Centro de Vigilância Epidemiológica "Prof. Alexandre Vranjac" - Secretaria de Estado da Saúde de São Paulo e Professor da Universidade São Judas Tadeu - São Paulo. https://orcid. org/0000-0002-0685-4627

\section{Ana Paula Miranda Mundim-Pombo}

Enfermeira e Médica Veterinária, Doutora em Ciências pela Faculdade de Medicina da Universidade de São Paulo, Professor da Universidade São Judas Tadeu - São Paulo. São Paulo (SP). https://orcid.org/00000002-1877-0965

Recebido em: 27/05/2020

Aprovado em: 01/07/2020

\section{Janessa de Fátima Morgado de Oliveira}

Farmacêutica-bioquímica, Doutora em Ciências pela Faculdade de Saúde Pública da Universidade de São Paulo, Investigadora - Departamento de Epidemiologia do Instituto de Saúde Pública da Universidade do Porto (Projeto DOCnet). Porto (Porto). https://orcid.org/0000-0002-8482-5872

INTRODUÇÃO

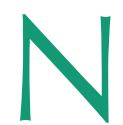
o mês de dezembro de 2019, no Município de Wuhan, província de Hubei, China, um surto de pneumonia de causa desconhecida desencadeou investigações epidemiológicas, de modo que em 7 de janeiro de 2020 foi isolado um novo vírus altamente contagioso, Severe Acute Respiratory Syndrome Coronavírus 2 (SARS-CoV-2), causador da síndrome respiratória aguda grave, COVID-19(1), e já em janeiro de 2020, a Organização Mundial da Saúde declarou o surto enquanto emergência de saúde pública de interesse internacional que, devido expansão em nível global, em março de 2020, foi declarada pandemia ${ }^{(2-4)}$.

A disseminação do agente se manteve até que em até 16 de maio de 2020 já haviam sido confirmados 4.425.285 casos de COVID-19 no mundo e 302.059 mortes. Essa casuística foi, principalmente, composta por notificações oriundas das regiões: europeia (1.848.445 casos/ 164.723 mortes); americana (1.909.483 casos/ 3.123 mortes); mediterrâneo oriental (315.688 casos/ 9.701 mortes); pacífico ocidental (166.721 casos/ 6.697 mortes), sudeste asiático (127.995 casos/ 4.201 mortes) e africana (54.461 casos/ 1.661 mortes) $)^{(4)}$. 
Nas Américas, chama a atenção à casuística apresentada pelo Brasil, uma vez que, em 16 de maio de 2020, já havia sido confirmados 233.142 casos e 15.633 mortes $^{(5)}$, tratando-se do principal país acometido na América Latina ${ }^{(4)}$. Ao considerar a taxa de incidência e de mortalidade de COVID-19 por 100.000 habitantes no país, no mesmo período, tem-se respectivamente 110,9 e 7,4, sendo que as duas principais regiões acometidas foram a norte e sudeste. Considerando ainda o período desde a confirmação do primeiro caso até 16 de maio de 2020, destaca-se a região sudeste por se apresentar como a segunda principal em termos de taxa de incidência e de mortalidade, sendo 106,2 e 8,7 , respectivamente ${ }^{(5)}$.

Destaca-se na região sudeste o Estado de São Paulo, onde ocorreu a notificação do primeiro caso no Brasil e América Latina, quando um brasileiro de 61 anos, após viagem de 9 a 20 de fevereiro de 2020 à Lombardia, região norte da Itália, foi confirmado com COVID-19(6). Até 16 de maio de 2020, nesta região, já haviam sido notificados 61.183 casos e 4.688 óbitos $^{(5)}$.

Em vigilância epidemiológica, a informação é fundamental para realização de ações em saúde coerentes com a realidade local, de forma que obter maior conhecimento sobre a distribuição e frequência da COVID-19 pode colaborar para o entendimento sobre a epidemiologia da doença, o que fundamenta o interesse em investigar mais detalhadamente esse problema em saúde pública no Estado de São Paulo. Diante do exposto o presente estudo teve como objetivo descrever a incidência, mortalidade e letalidade causadas pela nova doença, de 26 de fevereiro a 26 de abril de 2020, no Estado de São Paulo.

\section{MÉTODOS}

Estudo epidemiológico, do tipo ecológico, de base populacional. A população do Estado de São Paulo foi objeto do estudo, considerando sua distribuição nos 645 municípios do estado. Os casos confirmados do Novo Coronavírus (COVID-19) foram obtidos a partir da base de dados de domínio público do Governo do Estado de São Paulo - Secretaria de Estado da Saúde - Coordenadoria de Controle de Doenças - Centro de Vigilância Epidemiológica "Prof. Alexandre Vranjac" - Novo Coronavírus (COVID-19) - Situação Epidemiológica 26 de fevereiro a 26 de abril de 2020, disponíveis em: http://www.saude.sp. gov.br/cve-centro-de-vigilancia-epidemiologica-prof.-alexandre-vranjac/ areas-de-vigilancia/doencas-de-transmissao-respiratoria/coronavirus-covid-19/situacao-epidemiologica.

Para o cálculo das taxas de incidência e mortalidade, foi utilizada a população obtida do sítio eletrônico do Instituto Brasileiro de Geografia e Estatística (IBGE) 2019. As taxas de incidência e mortalidade por 100.000 habitantes foram calculadas a partir da razão entre o número de casos confirmados e óbitos, respectivamente (numerador) e a população residente (denominador). A letalidade por 100 casos foi calculada a partir da razão entre o número de óbitos (numerador) e os casos confirmados (denominador). Para a análise dos dados, utilizou-se estatística descritiva, aplicando-se os Softwares TabWin 3.6b e o Microsoft Excel 2016.

A pesquisa não precisou ser aprovada por Comitê de Ética de Pesquisa, haja vista que os dados secundários da COVID-19 obtidos para análise nesse estudo são de domínio público e não apresentou identificação dos pacientes, seguindo, assim, os princípios estabelecidos pela Resolução no 466, de 12 de dezembro de 2012, que dispõe sobre as diretrizes e normas regulamentadoras de pesquisa envolvendo seres humanos.

\section{RESULTADOS}

O primeiro caso de COVID-19 no Estado de São Paulo foi confirmado em 25 de fevereiro de 2020 no município de São Paulo. Após dois meses da epidemia por COVID-19 (26 de abril de 2020), o Estado de São Paulo já tinha 21.696 casos confirmados em 288 municípios (44,65\% dos 645 municípios), sendo o município de São Paulo o mais atingindo $(64,48 \%)$ (Figura 1$)$.

Figura 1 - Distribuição dos casos confirmados do Novo Coronavírus (2019-nCoV), segundo município de residência. Estado de São Paulo, 26 de fevereiro a 26 de abril de 2020.

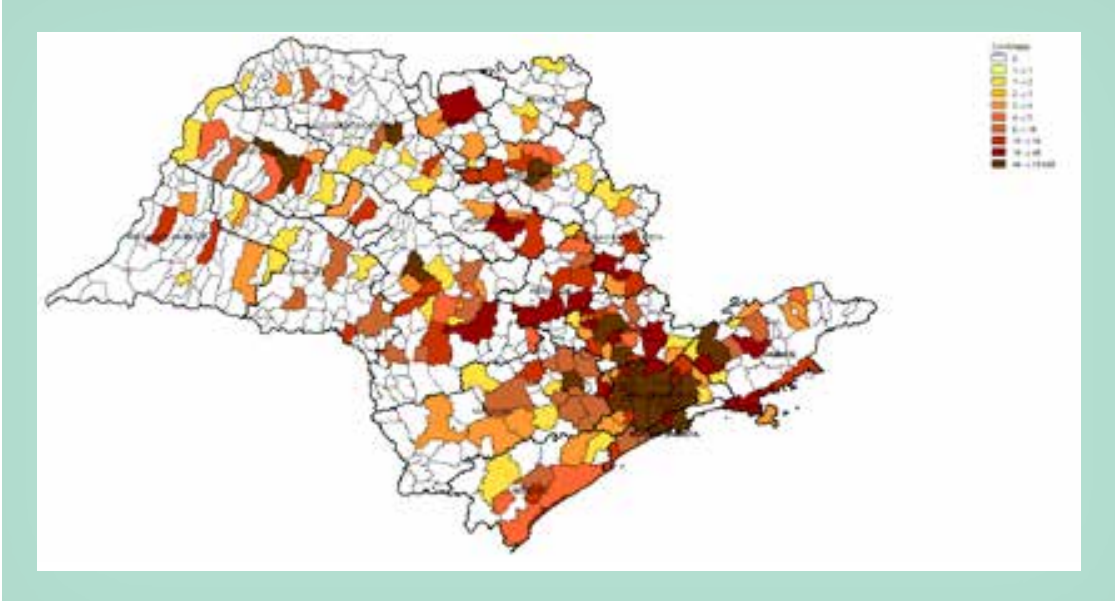

Fonte: Governo do Estado de São Paulo / Secretaria de Estado da Saúde / Coordenadoria de Controle de Doenças / Centro de Vigilância Epidemiológica "Prof. Alexandre Vranjac" - Novo Coronavírus (COVID-19) - Situação Epidemiológica 61 
Para o período estudado, o Estado de São Paulo apresentou a taxa de incidência de COVID-19 de 47,25 casos para cada 100.000 habitantes, sendo os municípios com maiores taxas: Arandu $(141,58$ casos para cada 100.000 habitantes), Ilha Comprida (116,42 casos para cada 100.000 habitantes), São Paulo $(114,18$ casos para cada 100.000 habitantes), Santos $(106,85$ casos para cada 100.000 habitantes) e Jaci $(113,20$ casos para cada 100.000 habitantes) (Figura 2).

O primeiro óbito por COVID-19 foi registrado em 17 de março de 2020 no município de São Paulo e em 26 de abril o Estado de São Paulo confirmou 1825 óbitos em 131 municípios (20,31\% dos 645 municípios). O município de São Paulo obteve o maior número de óbitos (64,22\%) (Figura 3).

A taxa de mortalidade de CO-
Figura 2 - Distribuição da Taxa de Incidência por 100.000 habitantes do Novo

Coronavírus (2019-nCoV), segundo município de residência. Estado de São Paulo, 26 de fevereiro a 26 de abril de 2020.

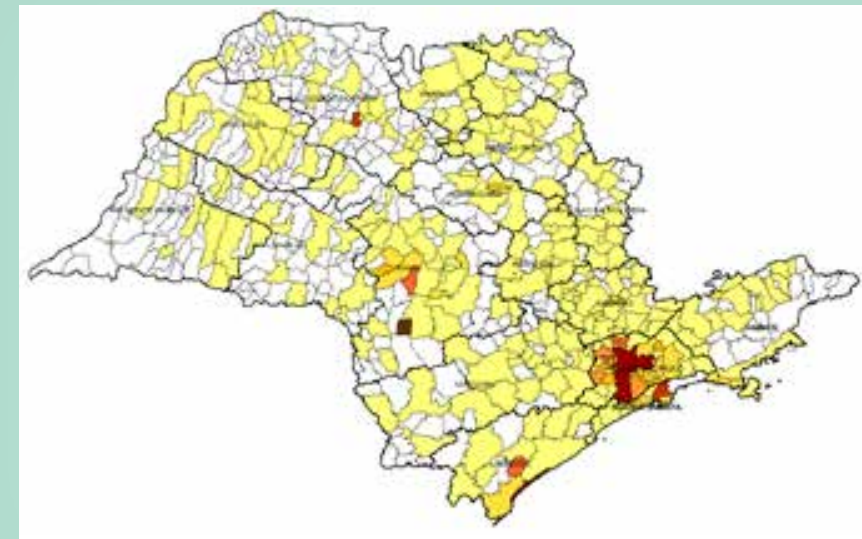

Fonte: Governo do Estado de São Paulo / Secretaria de Estado da Saúde / Coordenadoria de Controle de Doenças / Centro de Vigilância Epidemiológica "Prof. Alexandre Vranjac" - Novo Coronavírus (COVID-19) - Situação Epidemiológica 61

Figura 3 - Distribuição dos óbitos confirmados do Novo Coronavírus (2019-nCoV), segundo município de residência. Estado de São Paulo, 26 de fevereiro a 26 de abril de 2020.

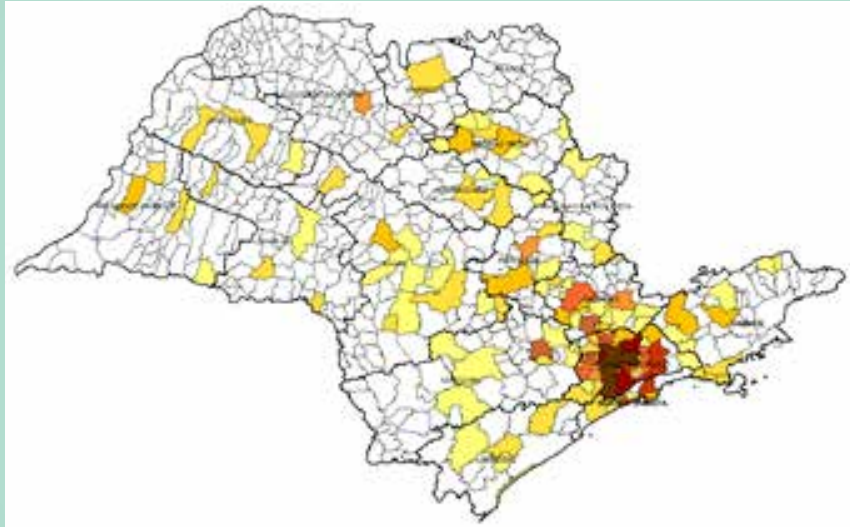

Fonte: Governo do Estado de São Paulo / Secretaria de Estado da Saúde / Coordenadoria de Controle de Doenças I Centro de Vigilância Epidemiológica "Prof. Alexandre Vranjac" - Novo Coronavírus (COVID-19) - Situação Epidemiológica 61
VID-19 no Estado de São Paulo foi de 3,97 casos para cada 100.000 habitantes, sendo os municípios de maiores taxas: Arandu $(31,46$ casos para cada 100.000 habitantes), Caiabu $(23,86$ casos para cada 100.000 habitantes), Presidente Venceslau $(15,18$ casos para cada 100.000 habitantes), Santo Antonio da Alegria $(14,43$ casos para cada 100.000 habitantes) e Lavrinhas $(13,77$ casos para cada 100.000 habitantes) (Figura 4).

A razão entre o número de óbitos e os casos confirmados (letalidade) por COVID-19 no Estado de São Paulo foi de 8,41 para cada 100 casos. Os municípios de Caiabu, Conchas, lepê, Jarinu, Juquitiba, Pitangueiras, Rincão, Salesópolis, Santa Rita do Passa Quatro, Santo Antônio da Alegria e Serrana apresentaram 100\% desse indicador (Figura 5).

\section{DISCUSSÃO}

A identificação de áreas potencialmente de risco para doenças infecciosas e dos fatores que influenciam na distribuição espacial delas é relevante para as autoridades de saúde pública para implementar medidas efetivas de prevenção e controle ${ }^{(7)}$. Com o propósito de descrever os padrões espaciais que possam ajudar na compreensão dos fatores que podem influir no risco de contrair a doença, foi conduzido um estudo ecológico que comparou a magnitude dos indicadores incidência, mortalidade e letalidade da epidemia do Novo Coronavírus (doença por COVID-19) entre os municípios do estado de São Paulo para o período de dois meses. Tais dados poderão servir de referência para comparação com os dados para o período de três meses, ou mais, para avaliação do impacto das medidas adotadas e da evolução da doença.

As taxas de incidência e de mortalidade para o município de São Paulo foram 114,2 e 9,6, respectivamente, bem mais altos que os observados em nível mundial. Considerando todos os casos 
Figura 4 - Distribuição da Taxa de Mortalidade por 100.000 habitantes do Novo Coronavírus (2019-nCoV), segundo município de residência. Estado de São Paulo, 26 de fevereiro a 26 de abril de 2020.

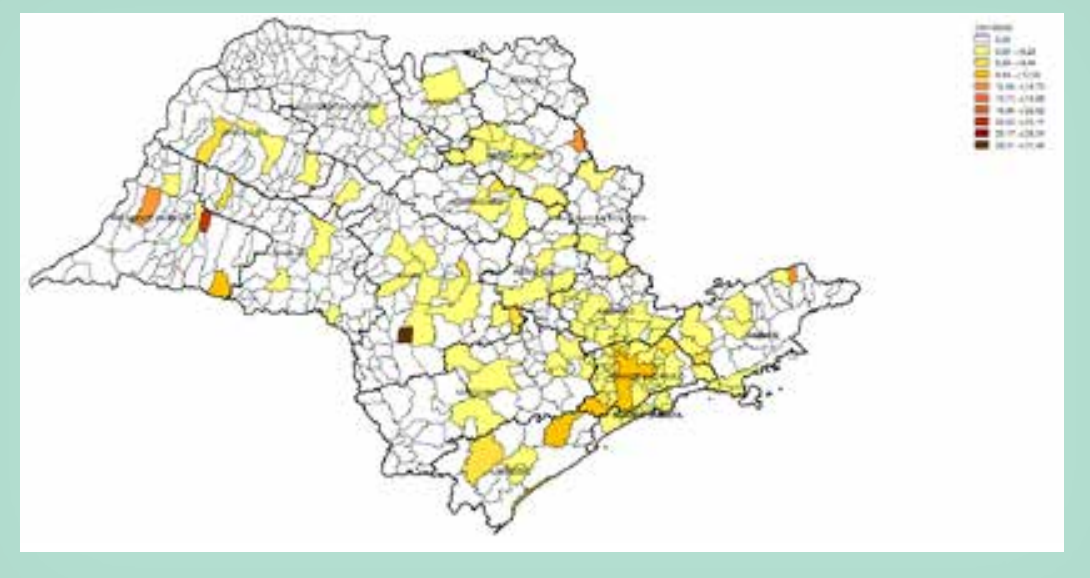

Fonte: Governo do Estado de São Paulo / Secretaria de Estado da Saúde / Coordenadoria de Controle de Doenças / Centro de Vigilância Epidemiológica "Prof. Alexandre Vranjac" - Novo Coronavírus (COVID-19) - Situação Epidemiológica 61

Figura 5 - Distribuição da Letalidade por 100 casos do Novo Coronavírus (2019nCoV), segundo município de residência. Estado de São Paulo, 26 de fevereiro a 26 de abril de 2020.

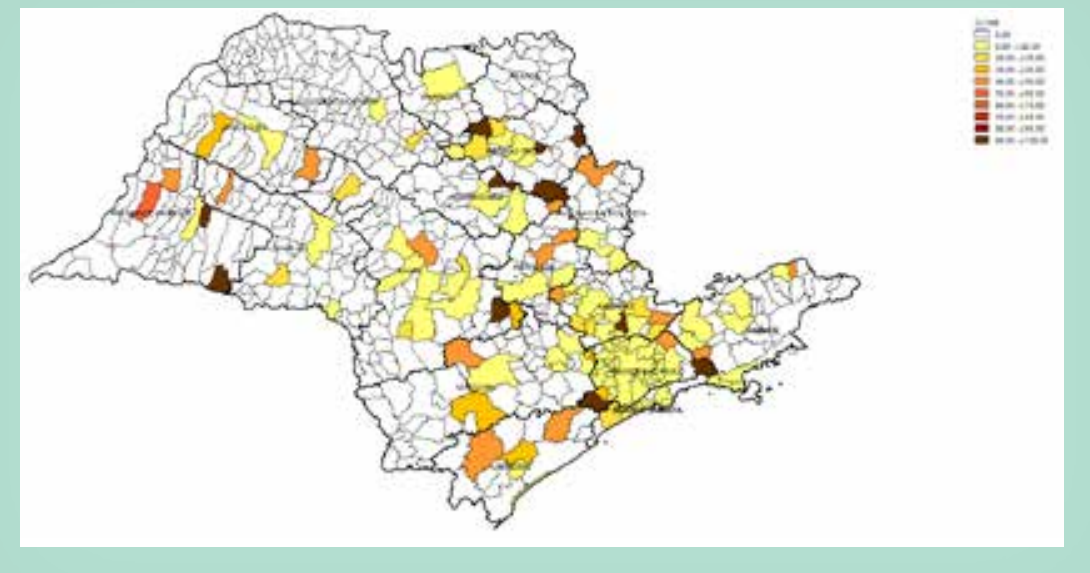

Fonte: Governo do Estado de São Paulo / Secretaria de Estado da Saúde / Coordenadoria de Controle de Doenças / Centro de Vigilância Epidemiológica "Prof. Alexandre Vranjac" - Novo Coronavírus (COVID-19) - Situação Epidemiológica 61

desde o início da pandemia até o dia 14 de maio, a taxa de incidência foi 57,4 casos para 100.000 habitantes e a de mortalidade foi de 3,8 óbitos para cada 100.000 habitantes ${ }^{(8)}$.

Devido à grande concentração populacional e às condições socioeconômicas mais díspares, as megacidades da China (Pequim, Guangzhou, Xanghai e Shenzhen) foram confrontadas com ten- sões maiores dos surtos de COVID-19. Essas megacidades não são apenas núcleos da economia da China, mas também exercem influências globais nas economias mundiais. Também, apresentam regiões com grande população flutuante, o que foi considerado um importante fator de influência para a disseminação da infecção ${ }^{(9)}$. O mesmo acontece com o município de São Paulo.
Foi observado que a distribuição dos casos nas megacidades chinesas foi caracterizada pela existência de clusters familiares de casos (Pequim e Guangzhou) e grande relevância dos casos importados no total observado (Guangzhou e Shenzhen). Pequim, Guangzhou e Shenzhen apresentaram padrões diferentes de distribuição de casos de COVID-19 e diferentes fatores influenciadores para tais padrões, que evidenciaram a importância dos fatores socioeconômicos, com destaque para a densidade populacional, o número de supermercados e de pontos de ônibus, assim como da migração diária de pessoas provindas das cidades vizinhas, justificados pela característica de transmissibilidade da doença de pessoa a pessoa ${ }^{(9)}$.

Os municípios próximos à capital, também, apresentaram incidência e mortalidade altos. Este padrão não foi observado para a letalidade. De acordo com a literatura, regiões vizinhas tendem a apresentar taxas mais semeIhantes do que as regiões distantes ${ }^{(10)}$. A movimentação da população entre as cidades integrantes da Grande São Paulo e os municípios vizinhos favorece a disseminação do agente, como já foi dito.

Considerando os municípios mais afastados da capital do estado, há diferenças entre os que apresentaram as maiores magnitudes para cada indicador estudado. Os municípios com maiores magnitudes para as taxas de incidência foram Arandu (141,6 casos para 100.000 habitantes - maior que o observado para a capital do estado), Jaci $(113,2$ casos para 100.000 habitantes), Borebi $(75,4$ casos para 100.000 habitantes) e Agudos (40,3 casos para 100.000 habitantes).

As maiores taxas de mortalidade foram observadas em Arandu (31,5 óbitos para cada 100.000 habitantes), Caiabu (23,9 óbitos para cada 100.000 habitantes), Presidente Venceslau (15,2 óbitos para cada 100.000 habitantes), 
Santo Antonio da Alegria (14,4 óbitos para cada 100.000 habitantes) e Lavrinhas (13,8 óbitos para cada 100.000 habitantes) - todas maiores que a observada para a capital do estado. De acordo com a publicação Indicadores Básicos para a Saúde no Brasil: conceitos e aplicações ${ }^{(11)}$, a taxa de mortalidade específica por doenças transmissíveis estima o risco de morte pela doença considerada na população total da área geográfica em questão em um determinado período de tempo. Retrata a incidência dessa doença em segmentos populacionais vulneráveis, associada às condições de desenvolvimento socioeconômico e de infraestrutura ambiental. Reflete, também, a efetividade de medidas de prevenção e controle, bem como as condições de diagnóstico e da assistência médica oferecida à população.

Arandu foi o município cujas magnitudes de incidência e de mortalidade foram as maiores do estado. Contava com 6.123 habitantes no último censo (população estimada de 6.357 em 2019). Seu IDH é 0,685, considerado médio. Em 2017, o salário médio mensal era de 1.9 salários mínimos e a proporção de pessoas com ocupação em relação à população total era de $17,1 \%$. Considerando domicílios com rendimentos mensais de até meio salário mínimo por pessoa, tinha 29,1\% da população nessas condições. O município contava apenas com dois estabelecimentos de saúde do Sistema Único de Saúde (SUS) e 20 leitos para internação em 2009(12). Os dados sugerem condições precárias de trabalho, renda e acesso à saúde. Entretanto, não podemos atribuir o impacto da doença na região a tais características do município, uma vez que o número de casos confirmados na região foi pequeno (nove). O fato de a população do município ser pequena parece ser o que melhor justifica os achados.

A letalidade variou entre $0,9 \%$ e $100 \%$. Um estudo ecológico conduzi-

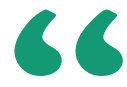

Sugere-se que a hipótese de que a letalidade seja tão mais alta nesses municípios, pela subestimação dos casos, seja investigada, pois, segundo publicação recente, são necessárias evidências sólidas (de boa qualidade) para combater as iniquidades em saúde durante o surto de COVID-19. do com dados de países com mais de 200 casos do novo coronavírus notificados analisou variáveis demográficas, de gasto sanitário e de características dos serviços de saúde como variáveis de exposição e as taxas de incidência, mortalidade e letalidade como variáveis de resposta ${ }^{(13)}$. O número de testes de diagnóstico da doença e o número de médicos tiveram associação com maior incidência da doença. Não foi verificada associação entre mortalidade e letalidade e as variáveis demográficas, de gasto com saúde ou dos serviços de saúde. Esses achados podem justificar as altas letalidades observadas em outros municípios do estado de São Paulo - em 24 deles, ela variou entre 54\% e $30 \%$ e em outros 20, entre $28 \%$ e $20 \%$ - pela investigação de doentes não ter sido realizada de forma mais ampla na população, ainda que o estudo citado tenha considerado localidades com mais de 200 casos da doença, o que não se aplica aos casos que tiveram altas letalidades no presente estudo.

Outra hipótese é que o número de casos da doença tenha sido muito pequeno e que os poucos casos tenham levado a óbito - o que pode justificar a letalidade de $100 \%$ em 11 municípios do estado. De acordo com os dados do World-o-Meter para o dia 14 de maio, a letalidade da doença no mundo é 6,7\% (4.471.418 casos confirmados no mundo e 299.612 óbitos). Sugere-se que a hipótese de que a letalidade seja tão mais alta nesses municípios, pela subestimação dos casos, seja investigada, pois, segundo publicação recente, são necessárias evidências sólidas (de boa qualidade) para combater as iniquidades em saúde durante o surto de COVID-19. Sem elas, a avaliação da equidade em saúde será impossível, o que reduzirá o impacto dos esforços de controle da doença ${ }^{(14)}$. Além disso, garantir oportunidades de diagnóstico e tratamento para todos é visto como um fator importante para vencer a batalha contra a doença ${ }^{(14)}$. 
Como já foi dito, o estudo é ecológico descritivo, conduzido com dados dos dois primeiros meses da epidemia no Estado. Também, é retrospectivo e a atualização dos dados pode apontar para uma situação epidemiológica diferente da descrita. A maior limitação da análise de dados ecológicos conduzida no presente estudo tem relação com a heterogeneidade entre os grupos estudados no nível de exposição à doença $^{(10)} \mathrm{e}$ aos fatores que influenciam seu impacto como problema de saúde pública, como a estrutura etária da população, a extensão da investigação de casos, o acesso em tempo oportuno a serviços de saúde, adoção de medidas de isolamento social e de outras medidas de redução do risco de contágio não serem conhecidos. Tais fatores não foram incluídos nas análises, haja vista a limitação da base de dados referida. Além disso, conduzir a comparação simples dos indicadores entre as regiões estudadas pode ser complicado, pois regiões com número de casos observados pequenos mostram grande variabilidade para as taxas estimadas ${ }^{(10)}$. Assim, as taxas mais altas tendem a ser observados para municípios pequenos,

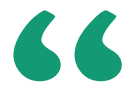

como pôde ser observado, provavelmente devido aos clusters de casos.

\section{CONCLUSÃO}

A capital do estado de São Paulo foi a região estudada mais atingida pela COVID19: 13989 casos confirmados e 1172 óbitos para o período estudado.
O potencial da epidemia em determinados municípios do estado de São Paulo é particularmente preocupante, dado ao grande número de pessoas potencialmente suscetíveis ao COVID19.

A capital do estado de São Paulo foi a região estudada mais atingida pela COVID19: 13989 casos confirmados e 1172 óbitos para o período estudado. No entanto, o município de São Paulo não apresentou maiores taxas de incidência, mortalidade e letalidade.

Os resultados deste estudo oferecem informações fundamentais para auxiliar os gestores em saúde pública na tomada de decisões, sensível ao tempo, nos níveis municipal e estadual, tendo em vista que o uso de mapas de taxas de incidência, mortalidade e letalidade podem ajudar em comparações temporais que permitam avaliar melhor a evolução da doença e os impactos das medidas adotadas, além de orientar decisões para a prevenção e controle do Novo Coronavírus (2019-nCoV).

\section{Referências}

1. Wang C, Horby PW, Hayden FG, Gao GF. A novel coronavirus outbreak of global health concern. The Lancet. 2020; 395 (10223). https://doi. org/10.1016/S0140-6736(20)30185-9

2. Jin Y, Yang H, Ji W, Wu W, Chen S, Zhan G, Duan G. Virology, Epidemiology, Pathogenesis, and Control of COVID-19. Viruses. 2020; 12(4): 372. Published 2020 Mar 27. doi:10.3390/v12040372

3. Di Gennaro F, Pizzol D, Marotta C, Antunes M, Racalbuto V, Veronese N, Smith L. Coronavirus deseases (COVID-19). Int J Environ Res Public Health. 2020; 17 (8): 2690. doi: 10.3390/ijerph17082690

4. World Health Organization. Coronavirus disease (COVID-19) Situation Report-117. 2020. Disponivel em: https://www.who.int/docs/default-source/ coronaviruse/situation-reports/20200516-covid-19-sitrep-117.pdf?sfvrsn=8f562cc_2. Acesso em 17/05/2020.

5. BRASIL, Ministério da Saúde, Secretaria de vigilância em saúde. Boletim Epidemiológico Especial-16. COE COVID-19. Brasília, 2020

6. Rodriguez-Morales AJ, Gallego V, Escalera-Antezana JP, Mendez CA, Zambrano LI, Franco-Paredes, et. al. COVID-19 in Latin America: The implications of the first confirmed case in Brazil. Travel Medicine and Infections desease. Disponivel em: https://doi.org/10.1016/j.tmaid.2020.101613. Acesso em: 17/05/2020.

7. Gao, X., Cao, Z. Meteorological conditions, elevation and land cover as predictors for the distribution analysis of visceral leishmaniasis in Sinkiang province, mainland China. Sci Total Environ. 2019; 646: 1111-6. doi: 10.1016/j.scitotenv.2018.07.391

8. World-o-Meter. Disponível em: https://www.worldometers.info/coronavirus/. Acesso em: 14 de maio de 2020.

9. Ren H, Zhao L, Zhang A, Song L, Liao Y, Lu W, Cui C. Early forecasting of the potential risk zones of COVID-19 in China's megacities. Science of The Total Environment. 2020; 729. https://doi.org/10.1016/j.scitotenv.2020.138995 10. Morgenstern H. Ecologic Studies in Epidemiology: Concepts, Principles and Methods. Annu Rev Public Health. 1995; 16: 61-81. doi: 10.1146/annurev.pu.16.050195.000425

11. OPAS. Organização Pan-americana de Saúde. REDE Interagencial de Informação para a Saúde - RIPSA. Indicadores Básicos para a Saúde no Brasil: conceitos e aplicações. 2. ed. - Brasília: Organização Pan-Americana da Saúde, 2008. p. 144. Disponível em: http://tabnet.datasus.gov.br/tabdata/ livroidb/2ed/CapituloC.pdf

12. IBGE. Instituto Brasileiro de Geografia e Estatística. Brasil. São Paulo. Arandu. Disponível em: https://cidades.ibge.gov.br/brasil/sp/arandu/pesquisa/37/30255

13. Figueiredo AM, Codina AD, de Figueiredo DCMM, Gil-García E, Kalache A. Letalidad del COVID-19: ausencia de patron epidemiológico. Gaceta Sanitaria (2020). doi: https://doi.org/10.1016/j.gaceta.2020.04.001

14. Wang Z, Tang K. Combating COVID-19: health equity matters. Nat Med. 2020 Apr; 26(4): 458. doi: 10.1038/s41591-020-0823-6. 